\title{
The influence of anisotropy in numerical modeling of orthogonal cutting of cortical bone
}

\author{
C. Santiuste ${ }^{\text {a,* }}$, M. Rodríguez-Millán ${ }^{\text {b }}$, E. Giner ${ }^{\mathrm{c}}$, H. Miguélez ${ }^{\mathrm{b}}$
}

\begin{abstract}
a Department of Continuum Mechanics and Structural Analysis, University Carlos III of Madrid, Avda. de la Universidad 30, 28911 Leganés, Madrid, Spain
${ }^{\mathrm{b}}$ Department of Mechanical Engineering, University Carlos III of Madrid, Avda. de la Universidad 30, 28911 Leganés, Madrid, Spain

${ }^{\mathrm{c}}$ Department of Mechanical and Materials Engineering (CITV), Universitat Politècnica de València, Camino de Vera, 46022 Valencia, Spain
\end{abstract}

\begin{abstract}
Cutting operations in bone are involved in surgical treatments in orthopaedics and traumatology. The importance of guaranteeing the absence of damage in the living workpiece is equivalent in this case to ensuring surface quality. The knowledge in this field is really far from the expertise in industrial cutting of mechanical components. Modeling of bone cutting is a challenge strongly dependent on the accurate modeling of mechanical behaviour of the bone. This paper focuses on modeling of orthogonal cutting of cortical bone. The intrinsic anisotropic nature of the cortical bone that makes it comparable to a compos-ite material is taken into account. The influence of anisotropy is analysed comparing this behaviour with an isotropic approach. It is shown that both chip morphology and temperature are affected by the anisot-ropy of the cortical bone that acts as a workpiece.
\end{abstract}

Keywords: Cortical bone, Orthogonal cutting, FE modeling, Temperature, Chip morphology.

\section{Introduction}

Cutting of bone is a common operation in orthopaedic/traumatologic surgery and dentistry. Cutting operations in bones involve a wide range of operations including grinding, sawing or drilling, see for instance [1-3]. It is well known in the field of manufacturing the importance of proper definition of machining operations for process efficiency (including both time and cost) and surface integrity. This concept is translatable to bone cutting since it is required to conjugate low cutting time (in order to diminish the total time of the surgery) and surface quality (mainly related with thermal bone damage) because the thermal affected layer at the machined bone could involve osteonecrosis [4].

The importance of the cutting processes for further evolution of the patient has motivated the study of bone machining. Thermal necrosis of the surrounding bone is the most important consequence of aggressive cutting of the bone. Osteonecrosis due to excessive temperature could cause further clinical problems such as loosening of bone-implant interface. Also grinding operations have received attention since they are commonly applied in intracranial surgery. The heated zone involves not only the bone, but also the blood vessel and nerves located close to the grinding zone [1]. Recent papers focus on the problem of osteonecrosis caused by

\footnotetext{
* Corresponding author. Tel.: +34 916249920; fax: +34 916249973.

E-mail address: csantius@ing.uc3m.es (C. Santiuste).
}

cutting bone during surgery. For instance, drilling is one of the most important operations and a summary of the state of the art is presented in $[4,5]$.

It is difficult to extract general conclusions from the works reviewed since the parameters involved in heat generation, such as the tool geometry, the cutting parameters or the use of coolant, are different in each application. The complexity of the problem makes the analysis of bone cutting very difficult with a purely experimental approach.

The use of validated modeling tools, such as the finite element method (FE) could help in the analysis and definition of machining operations in bones. However one of the most important factors in the achievement of accurate simulations is the statement of proper constitutive equations representative of bone behaviour.

Concerning the structure of the workpiece in surgery, it is worth that bones are composed of two main tissues: the cortical bone in the outer surface region and trabecular bone in the inner regions. The cortical bone is made of hard, dense tissues and takes charge of the main compressive and bending loads. The trabecular bones are made of sparse, rod-like tissue to reduce structural mass [6,7].

This paper focuses on cutting of cortical bone. In fact it is the first bone layer to be cut in any surgical operation. More specifically we concentrate on the case of diaphysis of long bones; it is important to note that significant differences in the structure can be found in other types of bone (for instance cranial). 
The damage in this part of the bone is critical considering its structural responsibility. In a micro-level (50-500 microns) the cortical bone is an anisotropic material suitable to be considered as a composite. It is possible to distinguish three relevant entities at this tissue level:

- Recent osteons they are cylinders (in a first approximation) with diameter ranging between 50-200 microns and length in the range $3-5 \mathrm{~mm}$. The osteons are formed in the continuous process of bone remodelling $[7,8]$.

- Interstitial matrix it is composed mainly of rests of old osteons with higher mineral content than recent osteons. The matrix presents lower toughness than recent osteons [7].

- Cement line it is a thin layer (about 1-5 microns [9]) surrounding the young osteons that appears during the formation of osteons. This zone exhibits low toughness, thus being a weak zone promoting crack propagation around the osteon $[9,10,26]$.

A simplified scheme of the cortical bone is shown in Fig. 1. A common assumption is to consider the cortical bone transversely isotropic, thus the mechanical properties in directions 2 and 3 would be identical but different of those in direction 1 (see Fig. 1).

Concerning the values of elastic properties it is possible to find contributions of different authors in the literature, mainly dealing with strength analysis of bone, since cutting has been poorly studied up to date. This fact will be further discussed in the following section.

Despite the anisotropic structure of bone, an isotropic approach in cutting bone modeling was presented in Alam et al. [11]. The authors carried out an experimental and numerical study focused on orthogonal cutting of bone. A two dimensional model of the process assuming elastic-viscoplastic behaviour of the bone for cutting forces and temperature prediction was presented. The mechanical response is represented by the Johnson-Cook law (without thermal softening). The Johnson-Cook model has been widely used for the simulation of metal cutting (see for instance recent works of the authors $[12,13])$. The same approach to the mechanical behaviour of bone was used in [14]. In this work a two dimensional model was applied to predict temperatures reached during bone drilling. Although the process is simplified considering orthogonal cutting and assuming an equivalency to cutting with the external side of the drill edge, reasonable accuracy is observed in temperature prediction.

Tu et al. [15] developed a model for drilling in the FE code ABAQUS explicit assuming an isotropic elastic-plastic behaviour of the bone, however no details concerning failure model were included in the paper. The influence of thrust force and cutting speed in temperature was obtained, finding decreasing temperature when both variables increased. Three dimensional approach required for drilling modeling involves rigorous simulation of rotation and feed movement of the tool (including penetration of the drill in the workpiece and element erosion) and usually leads to elevated computational cost. Lughmani et al. [16] developed a FE model in ABAQUS explicit for prediction of force and torque. In this work the bone was considered to be anisotropic and only elastic proper-ties are presented in the paper. Even in the case of carbon fibre composites, there are few works dealing with a complete approach to drilling and they have been recently developed $[17,18]$.

The detailed analysis of bone cutting is difficult using a 3D approach of the current operation, especially when the chip morphology is to be analysed. Orthogonal cutting has the advantage of allowing detailed simulation of the contact tool/chip and the use of small elements. Even in the case of metal cutting it is widely used to analyse the mechanisms involved in the cutting process.

The simulation of bone cutting has been poorly described in the scientific literature. This paper focuses on orthogonal cutting of cortical bone comparing an isotropic approach with an anisotropic behaviour of the bone which is assimilated to a composite material. In this work the simple case of orthogonal cutting is modelled with the aim of obtaining basic information concerning cutting forces, chip morphology and temperature, depending on material behaviour and cutting conditions. Two different models were developed: 2D for isotropic behaviour and 3D allowing the simulation of different orientations of the osteons. In the second model recent results obtained by the authors in composite cutting were considered, such as the influence of fibre orientation on chip morphology [19] and the prediction of temperature distribution [20]. Interesting conclusions were obtained, finding a strong influence of the anisotropy in chip morphology and temperature.

\section{Modeling behaviour of cortical bone}

Different models have been developed [11,21-28] with the aim of reproducing the behaviour of cortical bone. In this work, cortical bone of bovine femur is used as a reference and the numerical results are compared with the experimental data in the literature. The interest of this type of bone is related to its mechanical, fracture and thermal properties, very similar to those exhibited by human bone $[11,26]$. Two different models of cortical bone have been used: a rate dependent isotropic material and an anisotropic model dependent on the osteon orientation. Moreover, two different numerical models have been developed: 2D (for isotropic model of the bone) and 3D model allowing the simulation of the influence of osteon orientation.

The bone models are described in detail in the following subsections.

\subsection{Rate dependent isotropic material}

This model assumes von Mises J2 plasticity criteria based on a Johnson-Cook hardening law. Von Mises yield surface is defined by tension/compression symmetry. The Johnson-Cook hardening law is frequently applied to analyse the dynamic behaviour of

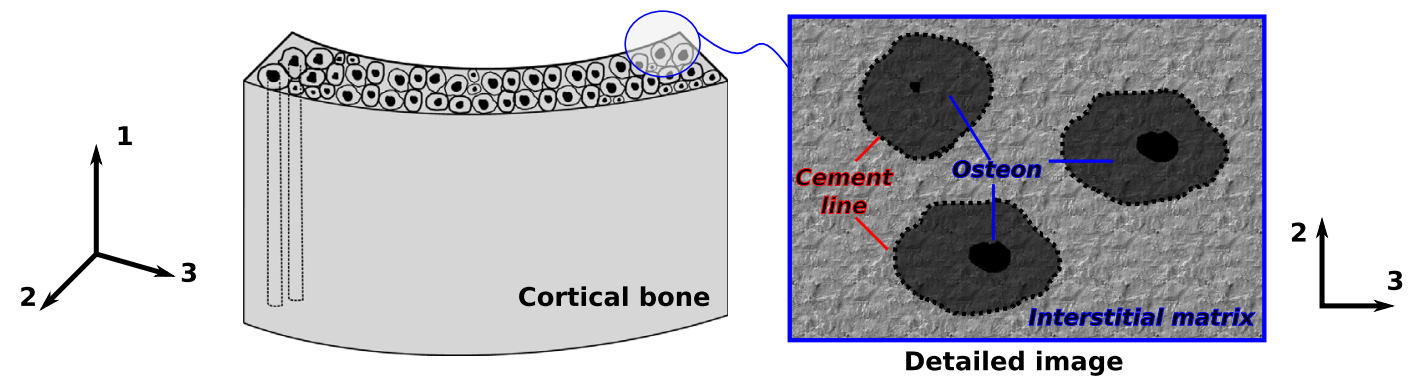

Fig. 1. Scheme of the cortical bone structure: Young osteons surrounded by cement line in an interstitial matrix composed by old osteons. 
metal alloys [29]. This hardening law is generally pre-implemented in FE codes, including ABAQUS/Explicit. The JC model is defined by Eq. (1). The first term defines strain hardening $\varepsilon^{-p}$, the second strain rate sensitivity, $\dot{\bar{\varepsilon}}^{p}$ via the constant $C$ and the third one is related to thermal softening $\Theta$, Eqs. (1) and (2).

$\bar{\sigma}\left(\bar{\varepsilon}^{p}, \dot{\bar{\varepsilon}}^{p}, T\right)=\left[A+B\left(\bar{\varepsilon}^{p}\right)^{n}\right]\left[1+C \ln \left(\frac{\dot{\bar{\varepsilon}}^{p}}{\dot{\bar{\varepsilon}}_{0}}\right)\right]\left[1-\Theta^{m}\right]$

$\Theta=\frac{T-T_{0}}{T_{m}-T_{0}}$

where $A$ and $B$ are material constants, $n$ is the strain hardening exponent, $m$ is the temperature sensitivity, $T_{0}$ is the initial temperature and $T_{m}$ is the melting temperature.

Keaveny et al. [32] obtained through experimental characterization of the bone its strain rate sensitivity at high strain rates, Fig. 2. Alam et al. [11] proposed to neglect the influence of temperature on yield stress due to small temperature changes leading to negli-gible thermal softening [14]. In this sense, Eq. (1) leads to Eq. (4):

$\bar{\sigma}\left(\overline{\bar{\varepsilon}}^{p}, \dot{\bar{\varepsilon}}^{p}\right)=\left[A+B\left(\overline{\bar{\varepsilon}}^{p}\right)^{n}\right]\left[1+C \ln \left(\frac{\dot{\bar{\varepsilon}}^{p}}{\dot{\bar{\varepsilon}}_{0}}\right)\right]$

These constants are reported in Table 1 corresponding to studies developed in the literature $[11,14,15]$.

\subsection{Anisotropic material}

Numerous studies have shown the anisotropic behaviour of bone, see e.g. [10,30-32]. The elastic modulus, yield stress and the ultimate stress change significantly with the stress direction (see Fig. 3, showing tensile behaviour of the bone dependent on loading direction). In Fig. 3 taken from [32], different samples were prepared considering several osteon directions with respect to the sample axis. The stiffness and strength are higher in the longitudinal direction (when the osteons direction coincides with the loading direction). This means that the assumption of mechanically isotropic bone tissue is a strong simplification of the problem. The cortical bone can be properly modelled as a rate dependent transversely isotropic material [33,34], thus the cortical bone tissue can be considered analogous to a fibre reinforced composite material [35-38]. Based on this analogy, the osteon acts as the fibre with the matrix being interstitial bone consisting of old osteon fragments. Numerous studies on fracture mechanics of cortical bone have demonstrated similarities to fracture mechanics in composite materials [35,39-43].

In this paper the anisotropic cortical bone was modelled assuming an elastic behaviour up to failure. Failure was predicted with

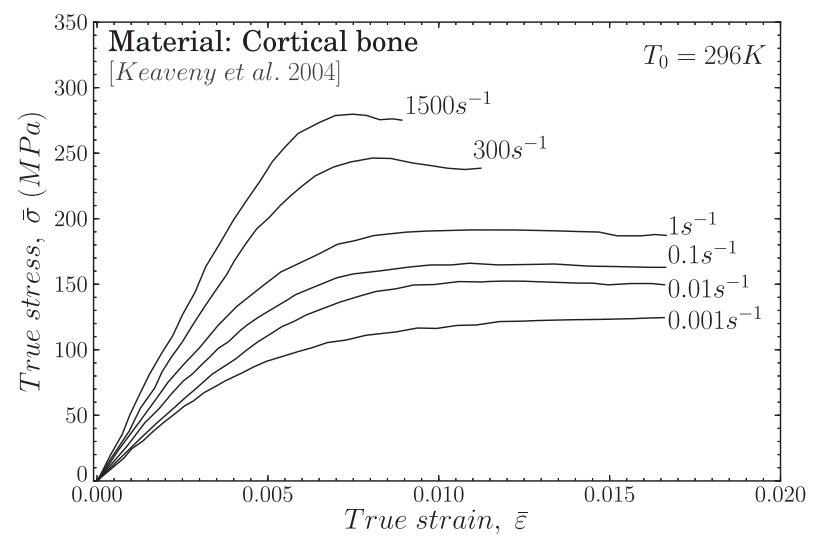

Fig. 2. Stress vs strain as a function of strain rate of bovine cortical bone at room temperature obtained from compression tests [11].
Table 1

Isotropic material properties and Johnson-Cook parameters used in cutting simulations $[11,14,15]$.

\begin{tabular}{llllll}
\hline Elastic and thermal properties & \multicolumn{5}{c}{ Plastic properties (JC model) } \\
\hline Modulus (GPa) & 20 & $\mathrm{~A}(\mathrm{MPa})$ & 50 & $\mathrm{C}$ & 0.03 \\
Poisson's ratio & 0.36 & $\mathrm{~B}(\mathrm{MPa})$ & 101 & $\dot{\varepsilon}_{0}\left(\mathrm{~s}^{-1}\right)$ & 0.001 \\
Density $\left(\mathrm{kg} / \mathrm{m}^{3}\right)$ & 2100 & $n$ & 0.08 & $T_{m}(\mathrm{~K})$ & 1573 \\
Thermal Conductivity $(\mathrm{W} / \mathrm{m} \mathrm{K})$ & 0.56 & $m$ & 0 & $T_{0}(\mathrm{~K})$ & 293 \\
Specific heat $(\mathrm{J} / \mathrm{Kg} \mathrm{K})$ & 1260 & & & & \\
\hline
\end{tabular}

Hou model [44] (Table 2). Hou approach, commonly used in composite mechanics, proposes the same formulation under tensile and compressive loading for fibre failure criterion, including longitudinal shear stresses $\sigma_{12}$ and $\sigma_{13}$. The transverse shear stress $\sigma_{23}$ was included for matrix cracking criterion. Parameters in Table 1 are the following: $\sigma_{11}, \sigma_{22}$, and $\sigma_{33}$, are the stresses in longitudinal, transverse and through-the-thickness direction respectively; $\sigma_{12}$, $\sigma_{23}$, and $\sigma_{13}$, are the shear stresses; $X_{T}$ and $X_{C}$ are the tensile and compressive strengths in longitudinal direction; $Y_{T}$ and $Y_{C}$ are the tensile and compressive strengths in the transverse direction; $S_{L}$ is the longitudinal shear strength; $S_{T}$ is the transverse shear strength. Failure occurs when any damage variable $\left(d_{i j}\right)$ reaches the value 1.

Hou formulation was implemented for the three dimensional analysis through a VUMAT user subroutine, developed to model fibre reinforced composite, including a procedure to degrade material properties (in this paper it is applied to the cortical bone behaviour). Under a given load, the stresses at each integration point were computed in the user subroutine. Then, each failure criterion was calculated as a function of stresses and, if any failure mode was reached, the material properties at that point were degraded according to the failure mode. When a failure criterion was verified in any element, the stresses were reduced to reproduce the properties degradation according to the failure mode.

The reduction of elastic properties could lead to distorted elements involving numerical problems, thus the model required the use of an element erosion criterion. The stresses on a damaged element drop to values close to zero while large deformations appear. These elements do not contribute to the strength or the stiffness of the plate, but they can cause lack of convergence during simulation and instability problems. Erosion criterion based on maximum strain criteria, was implemented in the VUMAT subroutine to remove the distorted elements. After each time increment the longitudinal strains $\left(\varepsilon_{11}, \varepsilon_{22}\right.$ and $\left.\varepsilon_{33}\right)$ were evaluated, and the element was removed if one of the strains reached a critical value. The strains used in the erosion criterion were $\varepsilon_{11}^{\max }=1$, $\varepsilon_{22}^{\max }=1$ and $\varepsilon_{33}^{\max }=1$, high enough to prevent the deletion of elements that contribute to the stiffness and strength of the workpiece. Thus, numerical problems were avoided and at the same time only strongly damaged elements were deleted. These values were established taking into account the capacity of the bone to experience deformation prior to rupture [45]. Since element deletion is controlled by the VUMAT user subroutine, distortion control was not used.

The parameters model were obtained from the work of Keaveny et al. [32] (see Table 3). These values are homogenised properties to be input in the numerical model.

Properties in direction 1 and 2 are the same order. This is a strong difference when comparing with structural composites such as CFRP which exhibit longitudinal stiffness two orders of magnitude higher than transversal. Concerning the strength properties, longitudinal values are higher both in tension and compression. Osteons are mainly aligned with the axis of the diaphysis of the long bone (although obviously some misalignment is found in the living tissue). The natural design imposes higher strength in the axis direction of the long bone than in the transverse direction 


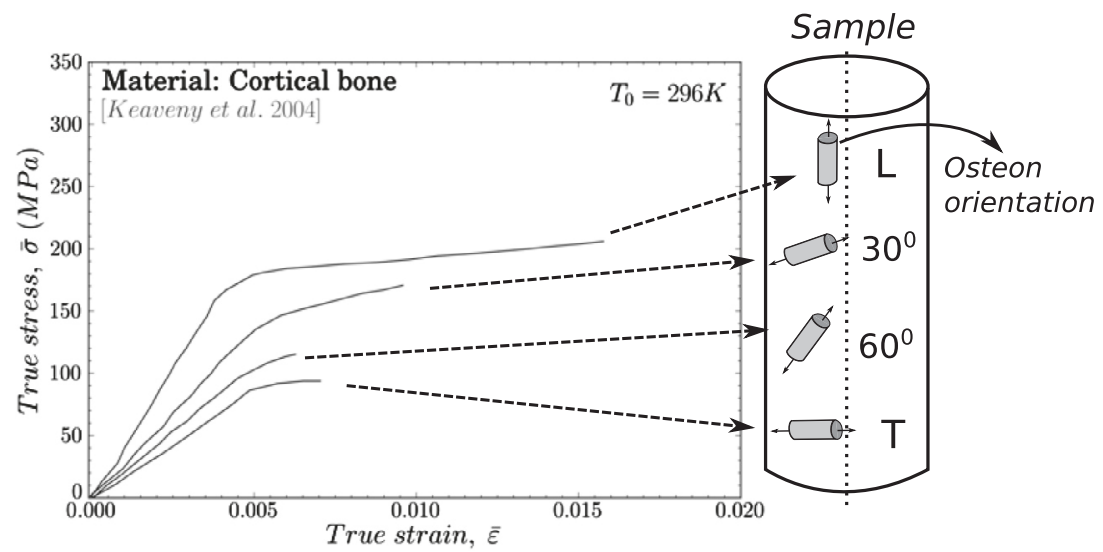

Fig. 3. Influence of osteon orientation on the mechanical behaviour of bone [32]. Small grey cylinder represents osteon orientation while the hollow cylinder represents the specimen used for compression tests.

Table 2

Hou damage criteria: failure modes for fibre and matrix.

\begin{tabular}{ll}
\hline Failure mode & Hou formulation \\
\hline Fiber tension & $d_{f t}^{2}=\left(\frac{\sigma_{11}}{X_{T}}\right)^{2}+\left(\frac{\sigma_{12}^{2}+\sigma_{13}^{2}}{S_{L}^{2}}\right)$ \\
Fiber compression & $d_{f c}^{2}=\left(\frac{\sigma_{11}}{X_{C}}\right)^{2}+\left(\frac{\sigma_{12}^{2}+\sigma_{13}^{2}}{S_{L}^{2}}\right)$ \\
Matrix cracking & $d_{m t}^{2}=\left(\frac{\sigma_{22}}{Y_{T}}\right)^{2}+\left(\frac{\sigma_{12}}{S_{L}}\right)^{2}+\left(\frac{\sigma_{23}}{S_{T}}\right)^{2}$ \\
Matrix crushing & $d_{m c}^{2}=\frac{1}{4}\left(\frac{-\sigma_{22}}{S_{T}}\right)^{2}+\left(\frac{Y_{c} \sigma_{22}}{4 S_{T}^{2}}\right)-\left(\frac{\sigma_{22}}{Y_{C}}\right)+\left(\frac{\sigma_{12}}{S_{L}}\right)^{2}$ \\
\hline
\end{tabular}

in order to deal with the usual loading state. On the other hand, compression behaviour is enhanced since the compressive loading in long bones is larger than tensile.

\section{Numerical model and validation}

The numerical modeling was developed using the commercial finite element code ABAQUS/Explicit with the objective of improving the understanding of mechanisms of chip generation. It is a useful tool in order to gather information on difficult to measure variables during machining processes and also diminishing the need of experimental work, which is commonly time consuming and expensive. Cutting parameters were stated in agreement with the experimental data in Alam et al. [14] used for models validation, Table 4 . The friction coefficient $(0.35)$ was set equal to the value used in [14] which was taken from the literature [46]. The initial temperature was stated equal to $37^{\circ} \mathrm{C}$ being approximately the normal temperature of a healthy adult human.

Two different models developed for orthogonal cutting are described in the following subsections:

\subsection{Bi-dimensional model for isotropic approach}

The bi-dimensional (2D) modeling of orthogonal cutting has been widely used in the scientific literature due to the simplicity
Table 4

Cutting parameters used in simulations of the bone cutting process.

\begin{tabular}{lc}
\hline Parameters & Magnitude used in ABAQUS \\
\hline Cutting speed, $V_{c}(\mathrm{~mm} / \mathrm{s})$ & $0.2,2,20$ \\
Friction coefficient & 0.35 \\
Tool nose radius $(\mu \mathrm{m})$ & 10 \\
Tool rake angle, $\gamma(\mathrm{deg})$ & 20 \\
DOC $(\mathrm{mm})$ & 0.1 \\
Bone initial temperature $\left({ }^{\circ} \mathrm{C}\right)$ & 37 \\
\hline
\end{tabular}

of the simulation (this is the approach used in [11,14] for bone cutting). A plane strain model was developed using the commercial finite element code ABAQUS/Explicit based on a Lagrangian formulation.

The scheme of the numerical model showing geometry and boundary conditions is presented in Fig. 4 . The workpiece is fixed in some boundaries and the cutting speed is applied to the tool. The geometry is defined according to $[11,14]$ being the references used for validation. A thermo-mechanical coupled analysis was developed by using 47,000 linear quadrilateral elements of type CPE4RT in ABAQUS notation [47] as illustrated in Fig. 4. Those are plane strain, quadrilateral, linearly interpolated, and thermally coupled elements with reduced integration and automatic hourglass control. The tool is defined as a rigid body in ABAQUS. The interaction between workpiece and tool was modelled using the contact algorithm surface-node surface available in ABAQUS/ Explicit [47].

\subsection{Three-dimensional model for anisotropic approach}

A 3D model has been developed in order to simulate orthogonal cutting process of anisotropic cortical bone. The scheme of the numerical model is shown in Fig. 5 including boundary conditions and geometry and the detail of the tool/chip interface.

Dynamic explicit analysis was carried out including geometric non-linearity and large deformations options. The tool was meshed

Table 3

Anisotropic properties of cortical bone [32].

\begin{tabular}{|c|c|c|c|c|}
\hline Elastic properties & & Ultimate stress & & \\
\hline Longitudinal modulus $\mathrm{E}_{1}(\mathrm{GPa})$ & 17.90 & Longitudinal (MPa) & & Shear (MPa) \\
\hline Transverse modulus $\mathrm{E}_{2}(\mathrm{GPa})$ & 10.1 & Tension, $X_{T}$ & 135 & 65 \\
\hline Shear modulus $\mathrm{G}_{12}(\mathrm{GPa})$ & 3.3 & Compression, $X_{C}$ & 202 & \\
\hline Longitudinal Poisson's ratio $v_{12}(-)$ & 0.4 & Transverse (MPa) & & \\
\hline \multirow[t]{2}{*}{ Transverse Poisson's ratio $v_{23}(-)$} & 0.4 & Tension, $Y_{T}$ & 53 & \\
\hline & & Compression, $Y_{C}$ & 131 & \\
\hline
\end{tabular}




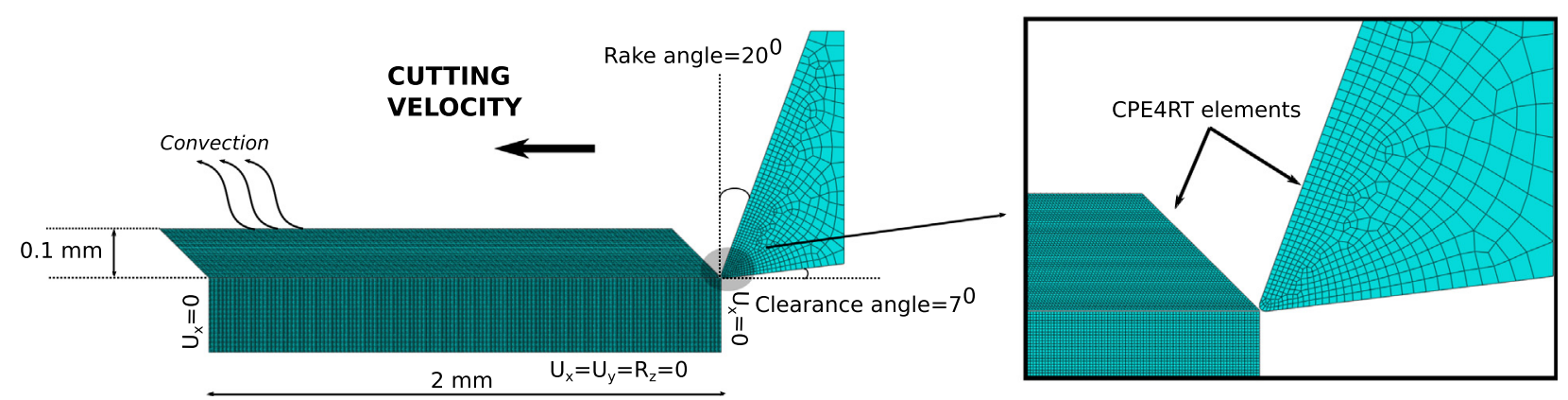

Fig. 4. Scheme of the 2D isotropic model for orthogonal cutting of bone.

with 8-node brick elements with reduced integration (C3D8R in ABAQUS/Explicit notation) and wedge elements (C3D6 in ABAQUS/Explicit notation [47]) were used for the workpiece. The use of wedge elements (prismatic element with triangular section) minimizes the dependence of the results with mesh orientation. A mesh sensibility analysis was carried out in previous work by the authors $[19,20])$. Therefore, it was set a minimum element size around the cutting zone equal to $7 \mu \mathrm{m}$ showing reasonably accuracy and computational cost.

The tool was assumed to behave as a rigid solid. The interaction between workpiece and tool was modelled using the contact algorithm surface-node surface available in ABAQUS/Explicit. The coefficient of friction at the tool-chip interface was 0.35 (the same value used in 2D model).

In order to analyse the influence of osteons orientation, three different cases: longitudinal, across and transversal have been carried out as illustrated in Fig. 6.

The same model validated through the comparison with experimental results provided in recent works dealing with orthogonal cutting of the carbon-epoxy LFRP composite T300/914 [48] was applied to the simulation of cortical bone cutting. Predicted cutting forces and temperature were compared with the values presented in Alam et al. [11] as described in next section.

\section{Numerical results: validation and discussion}

In this section the numerical results obtained with both developed models are presented. Validation of the models was performed through the comparison of predicted cutting forces and the forces measured in [11]. The numerical results predicted with isotropic and anisotropic models are compared, in terms of cutting forces, temperature and chip morphology. Significant differences are found, showing the importance of considering the anisotropy of the cortical bone.

\subsection{Cutting forces}

Fig. 7(a)-(d) presents the evolution of cutting force with cutting distance for both models analysed. Once the steady state is reached as the cutting progresses, the level of cutting force is close to the experimental value for the isotropic model. In the case of anisotropic model the mean value of the predicted force is in the order of the experimental value for the longitudinal orientation. The force increases for the across orientation. A similar behaviour was found for other composites CFRP and GFRP as it is shown in [49]. The orientation transversal to the plane also leads to increased values of the cutting force. Oscillations in the predicted force are due to the erosion of the elements.

These results are consistent with the trends found by different authors in the scientific literature [50,51] where cutting force presented the highest level in the transverse direction. Also Sui et al. [52] remarked the influence of the osteon orientation on the level of cutting force.

Concerning the influence of cutting speed on cutting force it is not possible to discriminate it with the anisotropic model since no rate dependence of material behaviour is considered. The isotropic model accounted for rate dependence through the implementation of JC constitutive law, however it is almost negligible as it is shown in Fig. 7d. This behaviour corroborates the experimental trends found by Yeager et al. [53].
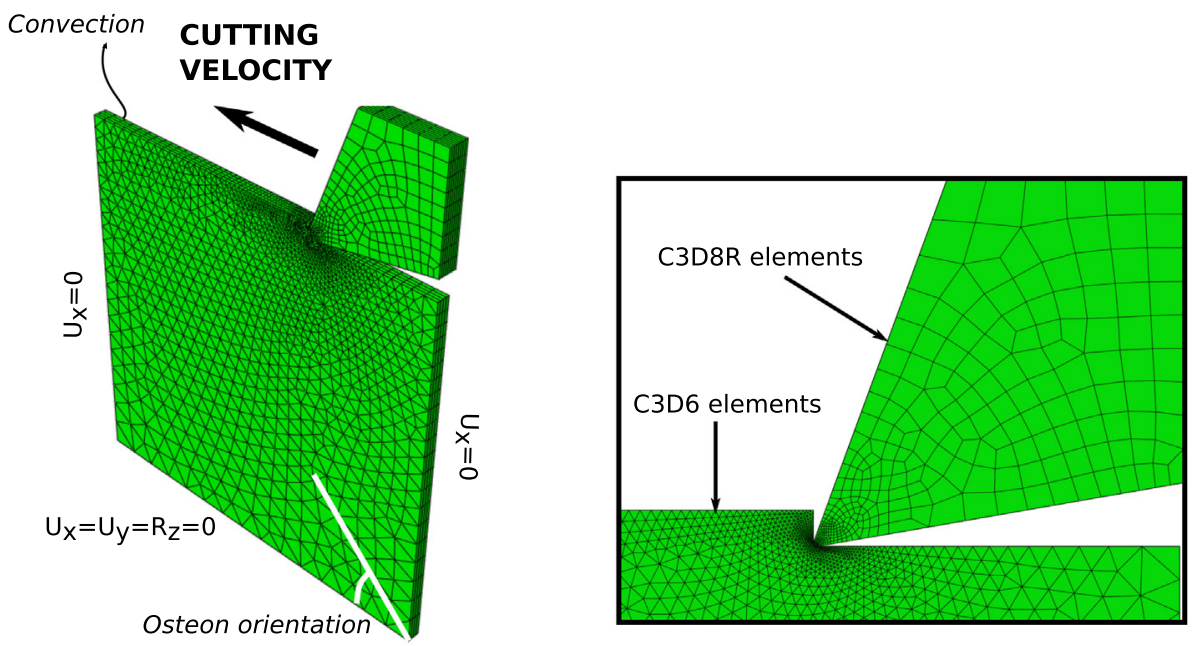

Fig. 5. Scheme of the numerical model for orthogonal cutting using a 3D model. 
a) Longitudinal

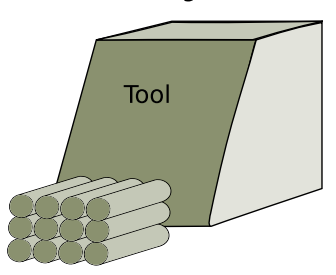

b) Across

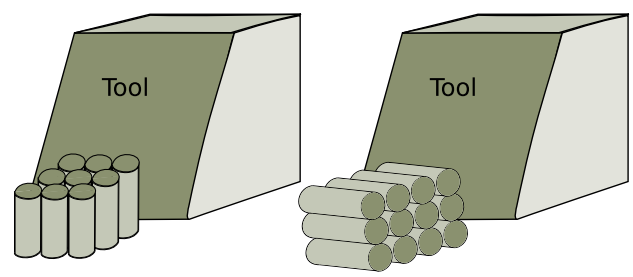

Fig. 6. Scheme of osteon orientation simulated in cutting process.

(a)

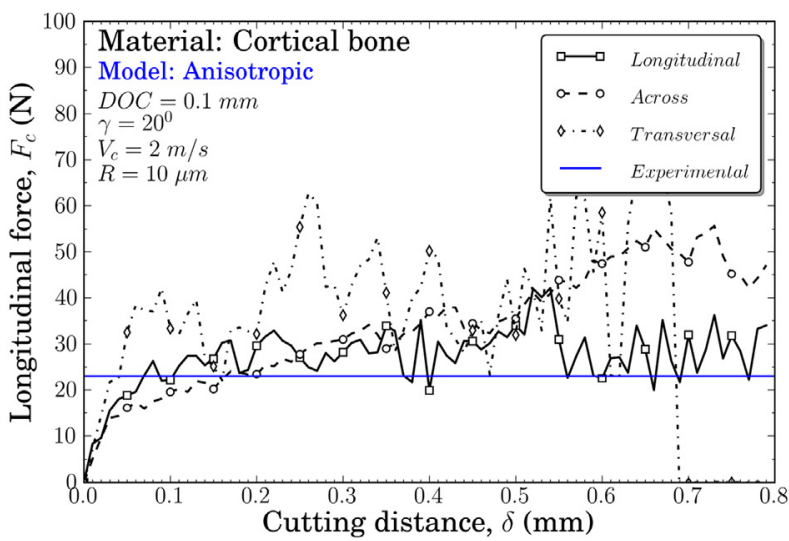

(c)

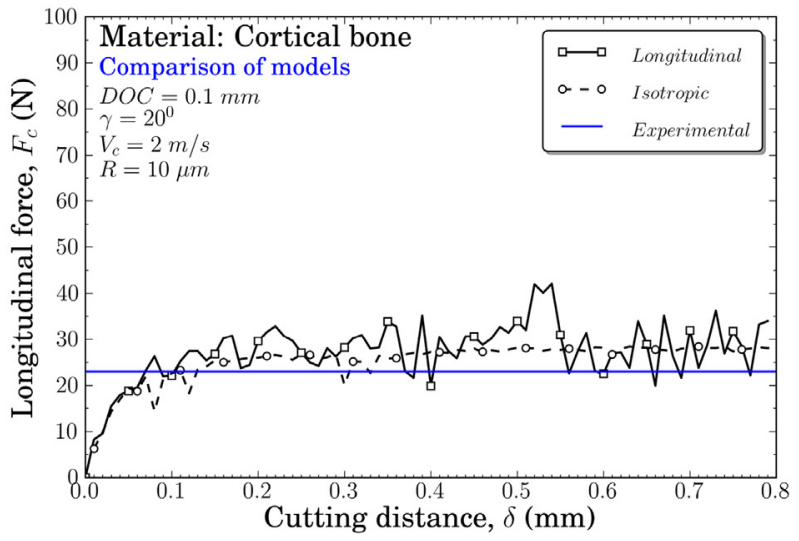

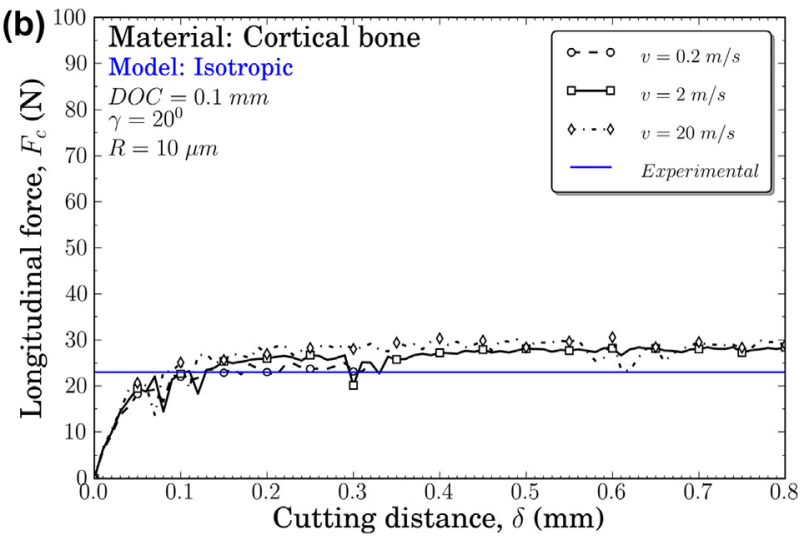

(d)

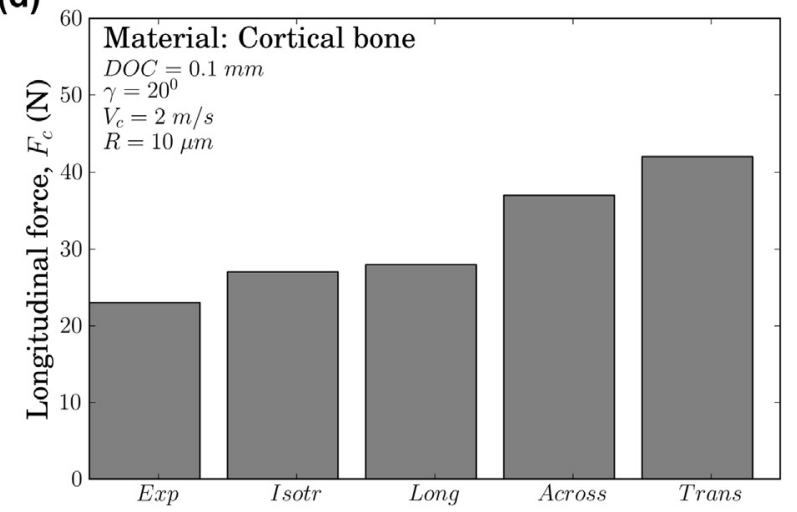

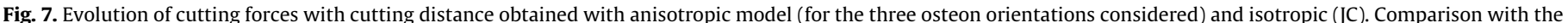

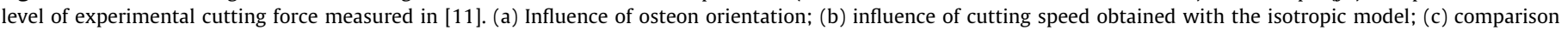

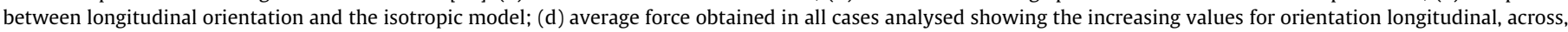
and transversal.

The influence of osteon orientation in the cutting forces is also described for other operations. Li et al. [27] developed experiments of cutting tool penetration in bone, the highest force was obtained when the osteons were orientated orthogonal to the indentation direction (equivalent to the transverse orientation described in the present work).

\subsection{Temperature and chip morphology}

As it has been commented previously the problem of temperature is related with osteonecrosis. Firstly the critical level of temperature should be established. There is some dispersion in the literature concerning the threshold below which osteonecrosis is avoided and thus the risk for the cell at the bone to be dead or damaged due to the exposure of high temperature. In this paper the critical level is stated equal to $47^{\circ} \mathrm{C}$ according to [15,22].
The contours of temperature are presented in Fig. 8, for the isotropic and anisotropic model with the different orientations considered, showing the highest temperature at the primary and secondary zones and also a thin layer thermally affected at the machined surface.

The influence of the constitutive model in chip morphology can be also observed in Fig. 8. Continuous chip is predicted with the isotropic model. Serrated chip is commonly due to adiabatic shear banding produced by thermal softening. However the isotropic JC model proposed in [11] does not account for thermal softening, thus serration is not predicted. The influence of the fibre (osteon) orientation is clear in Fig 8. Continuous chip is formed in the case of longitudinal orientation. Small chips due to the erosion are formed for the across orientation. And the triangular shaped chip is predicted for the transversal orientation, corresponding to the rupture of the interstitial matrix. 


\section{Temperature (K)}
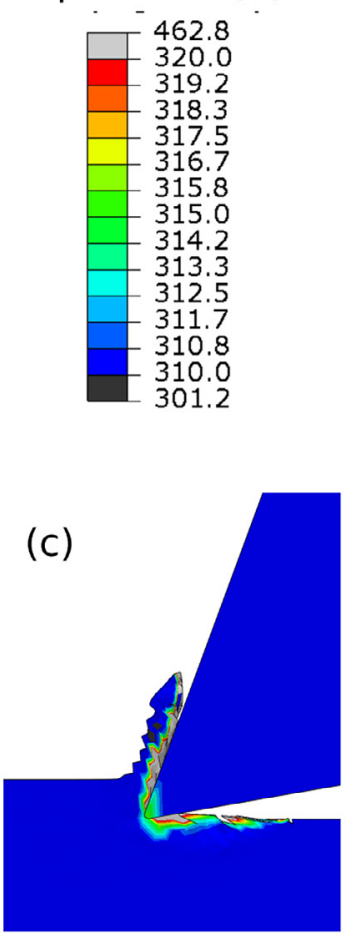
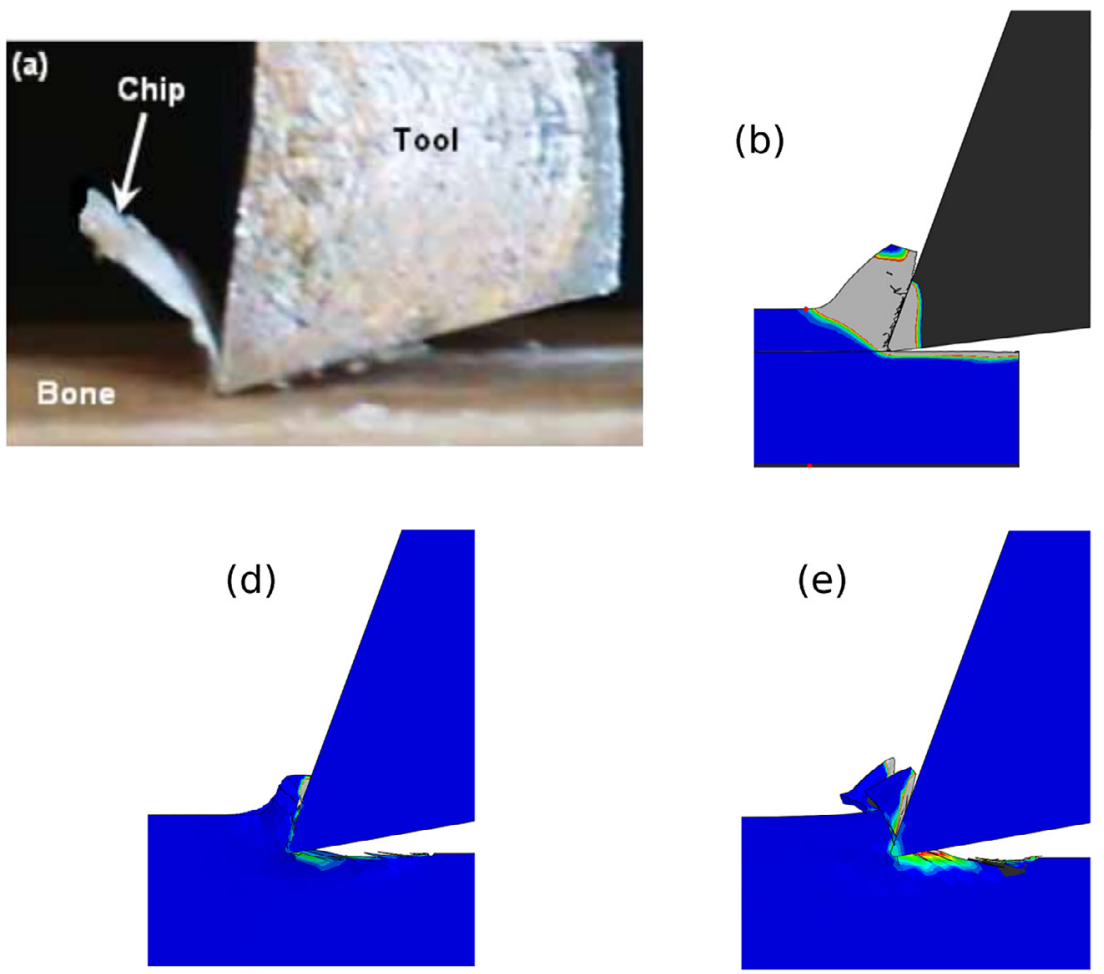

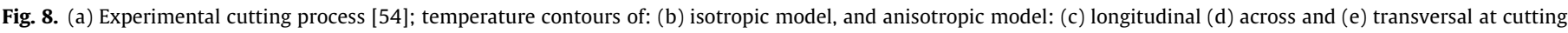
distance $=0.32 \mathrm{~mm}$.
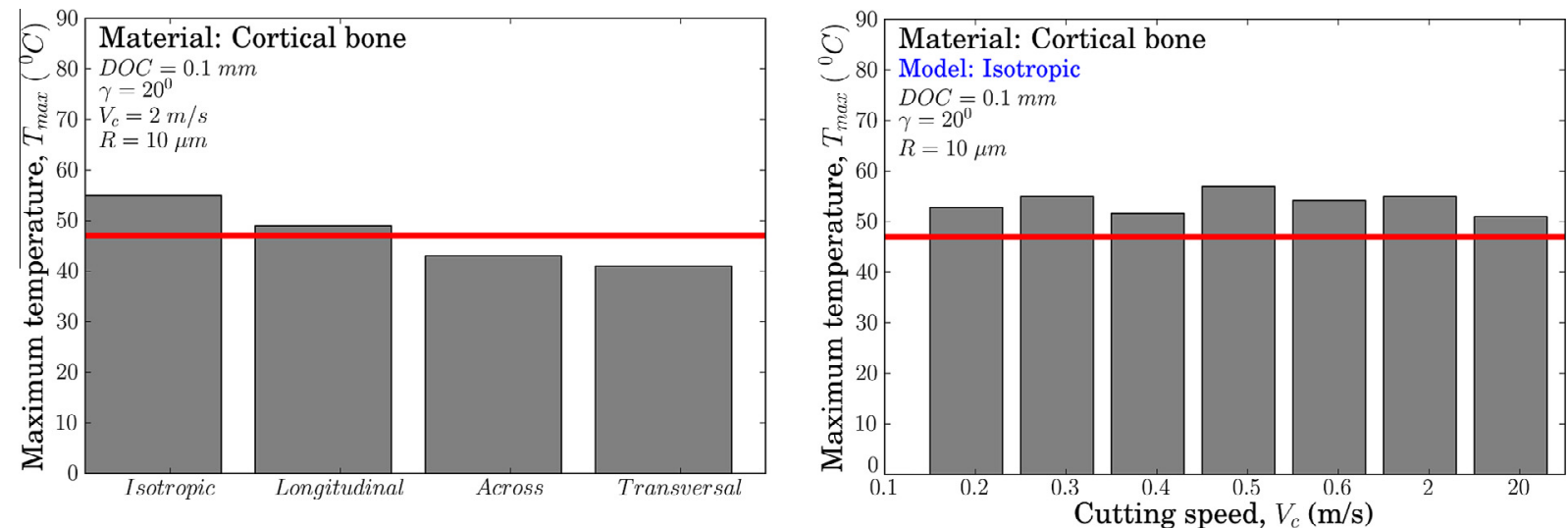

Fig. 9. Depth of thermally affected zone.
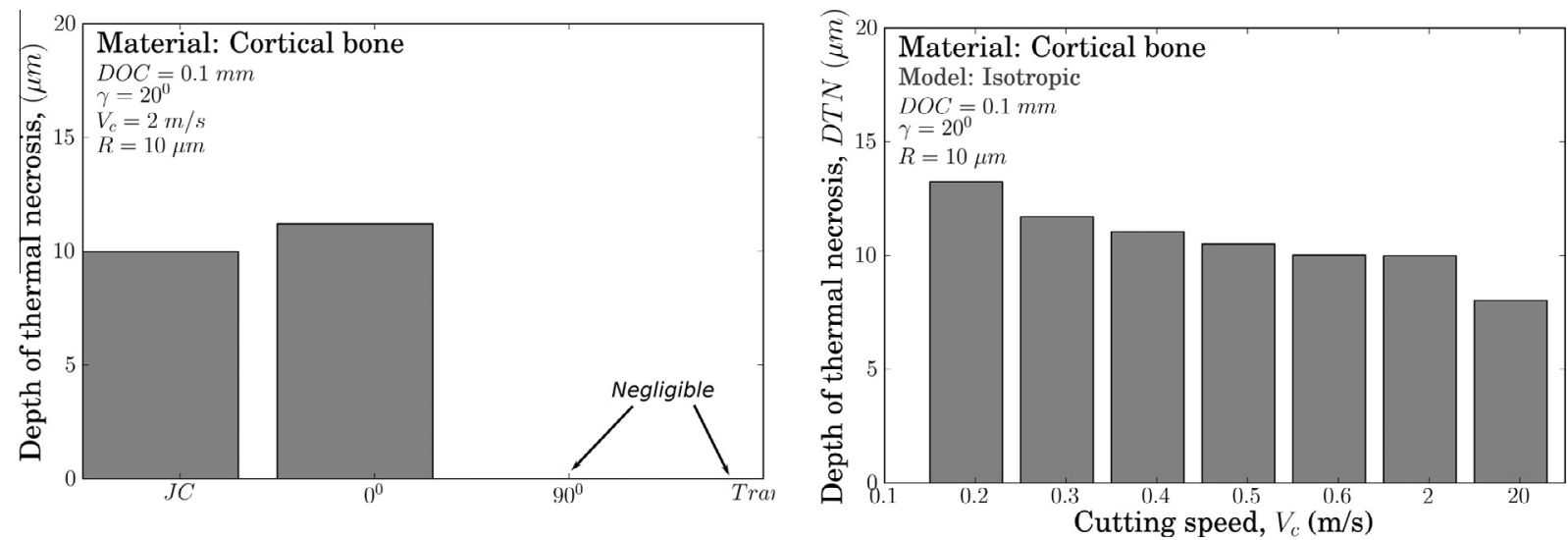

Fig. 10. Depth of thermal necrosis. 
The penetration of the thermally affected layer beneath the machined surface was obtained and it is presented in Fig. 9. The zone exhibiting temperature larger than $47^{\circ} \mathrm{C}$, i.e. the zone experiencing thermal necrosis, it is also highlighted in Fig 10.

The depth of thermally affected layer is lower for across and transversal orientations of the osteons. The critical threshold of temperature equal to $47^{\circ} \mathrm{C}$ is not reached for these orientations, however both longitudinal orientation and isotropic model reached temperatures higher than $47^{\circ} \mathrm{C}$. The depth of thermal necrosis for longitudinal orientation and isotropic model was found equal to 10 $\mu \mathrm{m}$ and $12 \mu \mathrm{m}$, respectively. These results show a great influence of chip morphology on the temperature reached in workpiece. Fig. 8 shows that the contact surface for longitudinal orientation and isotropic model is much higher than that of across and transversal orientation. Therefore, the heat generated due to friction forces is higher in the case of longitudinal orientation and isotropic model.

\section{Conclusions}

In this paper orthogonal cutting of cortical bone is analysed using a numerical approach based on finite elements. The bone is modelled as an isotropic material (using a constitutive model of Jonhson-Cook) and also as an anisotropic material using a similar approach to that used for long fibre reinforced composites. In this case the osteons play the role of the fibre reinforcing the interstitial matrix. The models commonly used in the literature for the simulation of metal and composite cutting were validated through the comparison with experimental results in $[11,14]$.

The influence of the osteon orientation on the cutting force is remarkable. The orientations across and transversal to the cutting speed direction, lead to the maximum levels of force. Moreover, this behaviour commonly observed experimentally in the literature has been reproduced with the anisotropic model developed.

The thermally affected zone also depends on osteon orientation. The level of $47^{\circ} \mathrm{C}$ corresponding with osteonecrosis was reached for the isotropic model and also for the longitudinal orientation, however the across and transversal orientation showed lower values of temperature.

The chip morphology strongly depends on the osteon orientation, changing from continuous chip similar to that obtained with the JC model for longitudinal orientation, to serrated chip for the transverse orientation.

\section{Acknowledgements}

The authors acknowledge the financial support for the work to the Ministry of Economy and Competitiveness of Spain under the Project DPI2011-25999 and DPI2013-46643-R.

\section{References}

[1] Tai BL, Zhang L, Wang A, Sullivan S, Shih AJ. Neurosurgical bone grinding temperature monitoring. Procedia CIRP 2013;5:226-30.

[2] James TP, Chang G, Micucci S, Sagar A, Smith EL, Cassidy C. Effect of applied force and blade speed on histopathology of bone during resection by sagittal saw. Med Eng Phys 2014;36(3):364-70.

[3] Fox MJ, Scarvell JM, Smith PN, Kalyanasundaram S, Stachurski ZH. Lateral drill holes decrease strength of the femur: an observational study using finite element and experimental analyses. J Orthop Surg Res 2013:8-29.

[4] Augustin G, Zigman T, Davila S, Udilljak T, Staroveski T, Brezak D, et al. Cortical bone drilling and thermal osteonecrosis. Clin Biomech 2012;27:313-25.

[5] Pandey RK, Panda SS. Drilling of bone: a comprehensive review. J Clin Orthop Trauma 2013;4:15-30.

[6] Kim SH, Chang SH, Jung HJ. The finite element analysis of a fractured tibia applied by composite bone plates considering contact conditions and timevarying properties of curing tissues. Compos Struct 2010;92:2109-18.

[7] Cowin SC, editor. Bone mechanics handbook. Boca Raton, Florida: CRC Press; 2001.
[8] Taylor D, Hazenberg JG, Lee TC. Living with cracks: damage and repair in human bone. Nat Mater 2007;6:263-8.

[9] Nobakhti S, Limbert G, Thurner PJ. Fracture toughness and mechanotransduction. J Mech Behav Biomed Mater 2014;29:235-51.

[10] Vercher A, Giner E, Arango C, Tarancón JE, Fuenmayor FJ. Homogenized stiffness matrices for mineralized collagen fibrils and lamellar bone using unit cell finite element models. Biomech Model Mechanobiol 2014;13(2):437-49.

[11] Alam K, Mitrofanov AV, Silberschmidt VV. Finite element analysis of forces of plane cutting of cortical bone. Comput Mater Sci 2009;46:738-43.

[12] Miguélez MH, Soldani X, Molinari A. Analysis of adiabatic shear banding in orthogonal cutting of Ti alloy. Int J Mech Sci 2013;75:212-22.

[13] Molinari A, Soldani X, Miguélez MH. Adiabatic shear banding and scaling laws in chip formation with application to cutting of Ti-6Al-4V. J Mech Phys Solids 2013;61:2331-59.

[14] Alam K, Mitrofanov AV, Silberschmidt VV. Thermal analysis of orthogonal cutting of cortical bone using finite element simulations. Int J Exp Comput Biomech 2010;1(3):236-51.

[15] Tu Y, Lu W, Chen L, Chiang C, Chen Y. Thermal contact simulation of drill bit and bone during drilling. In: Bioinformatics and Biomedical Engineerin (iCBBE), Proceedings of the 4th International Conference on, Chengdu, 18-20 June 2010. Vol. 6. p. 1-4.

[16] Lughmani WA, Bouazza-Marouf K, Ashcroft I. Finite element modeling and experimentation of bone drilling forces. J Phys Conf Ser 2013;451:012034.

[17] Isbilir O, Ghassemieh E. Numerical investigation of the effects of drill geometry on drilling induced delamination of carbon fiber reinforced composites. Compos Struct 2013;105:126-33.

[18] Feito N, López-Puente J, Santiuste C, Miguélez MH. Numerical prediction of delamination in CFRP drilling. Compos Struct 2014;108:677-83.

[19] Santiuste C, Miguélez MH, Soldani X. Out-of-plane failure mechanisms in LFRP composite cutting Compos Struct 2011:93:2706-13.

[20] Santiuste C, Díaz-Álvarez J, Soldani X, Miguélez MH. Modelling thermal effects in machining of carbon fiber reinforced polymer composites. J Reinf Plast Compos 2014;33(8):758-66.

[21] Davidson S, James DF. Drilling in bone: modeling heat generation and temperature distribution. J Biomech Eng 2003;125:305-14.

22] Sezek S, Aksakal B, Karaca F. Influence of drill parameters on bone temperature and necrosis: a FEM modeling and in vitro experiments. Comput Mater Sci 2012;60:13-8

[23] Basiaga M, Paszenda Z, Szewczenko J, Kaczmarek M. Numerical and experimental analyses of drills used in osteosynthesis. Acta Bioeng Biomech 2011;13:29-36.

[24] Kasiri S, Reilly G, Taylor D. Wedge indentation fracture of cortical bone: experimental data and predictions. J Biomech Eng 2010;132(8):1-6.

[25] Abdel-Wahab AA, Alam K, Silberschmidt VV. Analysis of anisotropic viscoelastoplastic properties of cortical bone tissues. J Mech Behav Biomed Mater 2010;4:807-20

26] Li S, Abdel-Wahab A, Demirci E, Silberschmidt VV. Fracture process in cortica bone: X-FEM analysis of microstructured models. Int J Fract 2013:184(12):43-55.

[27] Li S, Abdel-Wahab A, Demirci E, Silberschmidt VV. Penetration of cutting tool into cortical bone: experimental and numerical investigation of anisotropic mechanical behaviour. J Biomech 2013:1-10

[28] Vashishth D, Tanner KE, Bonfield W. Cortical bone during crack propagation. J Biomech 2000;33:1169-74.

[29] Johnson GR, Cook WH. A constitutive model and data for metals subjected to large strains high strain rates and high temperatures. In: Proceedings of the seventh international symposium on ballistics, 1983.

[30] Nordin M, Frankel VH. In: Nordin M, Frankel VH, editors. Basic biomechanics of the musculoskeletal system,. Lippincott Williams \& Wilkins; 2001.

[31] O'Mahony AM, Williams JL, Spencer P. Anisotropic elasticity of cortical and cancellous bone in the posterior mandible increases peri-implant stress and strain under oblique loading. Clin Oral Implant Res 2001;12(6):648-57.

[32] Keaveny TM, Morgan EF, Yeh OC. Bone mechanics. In: Standard handbook of biomedical engineering and design, 2004. p. 1-24.

[33] Reilly DT, Burstein AH. The elastic and ultimate properties of compact bone tissue. J Biomech 1975;8:393-405.

[34] Yoon H, Katz J. Ultrasonic wave propagation in human cortical bone. II. Measurements of elastic properties and microhardness. J Biomech 1976;9:459-64.

[35] Guo XE, Liang LC, Goldstein SA. Micromechanics of osteonal cortical bone fracture. J Biomech Eng 1998;120:112-7.

[36] Hogan HA. Micromechanics modeling of Haversian cortical bone properties. J Biomech 1992;25:549-56.

[37] Katz JL, Yoon HS, Lipson S, Maharidge R, Meunier A, Christel P. The effects of remodeling on the elastic properties of bone. Calcif Tissue Int 1984;36:31-6.

[38] Bigley RF, Griffin LV, Christensen L, Vandenbosch R. Osteon interfacial strength and histomorphometry of equine cortical bone. J Biomech 2006;39(9): 1629-40.

[39] Alto A, Pope MH. On the fracture toughness of equine metacarpi. J Biomech 1979;12:415-21.

[40] Bell KL, Loveridge N, Power J, Garrahan N, Meggitt BF, Reeve J. Regional differences in cortical porosity in the fractured femoral neck. Bone 1999;24:57-64.

[41] Martin RB, Gibson VA, Stover SM, Gibeling JC, Griffin LV. Osteonal structure in the equine third metacarpus. Bone 1996;19:165-71. 
[42] Moyle DD, Welborn 3rd JW, Cooke FW. Work to fracture of canine femoral bone. J Biomech 1978;11:435-40.

[43] Giner E, Arango C, Vercher A, Fuenmayor FJ. Numerical modeling of the mechanical behaviour of an osteon with microcracks. J Mech Behav Biomed Mater 2014;37:109-24.

[44] Hou JP, Petrinic N, Ruiz C, Hallett SR. Prediction of impact damage in composite plates. Compos Sci Technol 2000;60:228-73.

[45] Li S, Demirci E, Silberschmidt VV. Variability and anisotropy of mechanical behavior of cortical bone in tension and compression. J Mech Behav Biomed Mater 2013;21:109-20.

[46] Huang H, Chen S, Tang J. Analyses of rotating disc cutting of wood. Taiwan J For Sci 2003;18:263-71.

[47] Dassault Systèmes. Abaqus v6.12 Documentation- ABAQUS analysis user's manual. ABAQUS Inc; 6.12, 2012.

[48] Iliescu D, Gehin D, Iordanoff I, Girot F, Gutiérrez ME. A discrete element method for the simulation of CFRP cutting. Compos Sci Technol 2010;70:73-80.
[49] Santiuste C, Soldani X, Miguélez H. Machining FEM model of long fiber composites for aeronautical components. Compos Struct 2010;92:691-8.

[50] Jacobs $\mathrm{CH}$, Pope $\mathrm{MH}$, Berry JT, Hoaglund F. A study of the bone machining process - orthogonal cutting. J Biomech 1974;7(2):131-6.

[51] Wiggins KL, Malkin S. Orthogonal machining of bone. J Biomech Eng 1978;100(3):122-30.

[52] Sui J, Sugita N, Ishii K, Harada K, Mitsuishi M. Force analysis of orthogonal cutting of bovine cortical bone. Mach Sci Technol 2013;17(4):637-49.

[53] Yeager C, Nazari S, Arola D. Machining of cortical bone: surface texture, surface integrity and cutting forces. Mach Sci Technol 2008;12(1):100-18.

[54] Alam, K. Experimental and numerical analysis of convectional and ultrasonically assisted cutting of bone [thesis]. Loughborough University: 2009. p. 160. 